\title{
UN RADIOPROTECTEUR NON TOXIQUE ET ACTIF PAR VOIE ORALE LE : "SHIR-KHECHT»
}

\author{
G. MARBLE* et N. ROUHANIZADEH** \\ (manuscrit reçu le 23 mai 1967) $^{26}$
}

\begin{abstract}
SOMMAIRE
Le Shir-Khecht est une manne récoltée dans les environs de Téhéran. Ce produit, utilisé soit brut, soit après hydrolyse, purifié ou non par dialyse, présente une réelle activité radioprotectrice. Si on l'administre à des rats irradiés à dose létale, on peut observer un pourcentage de survie pouvant atteindre $50 \%$. L'effet protecteur est également observable à dose sublétale. Le grand intérêt du ShirKhecht réside dans le fait qu'il constitue un radioprotecteur d'origine naturelle, non toxique et qu'il est actif par voie orale.
\end{abstract}

\section{NATURE DU SHIR-KHECht}

Le Shir-Khecht est une manne récoltée dans les environs de Téhéran (Iran). Cette manne serait produite par une polygonacée, l'(Atraphaxis spinosa », selon Moghadam et plus vraisemblablement par une rosacée, le "Cotoneaster nummularia ", d'après PARSA. En fait, le produit brut peut être un mélange de mannes des deux origines. De composition chimique complexe (sucres, gommes, protéines, etc.) le Shir-Khecht est utilisé, en Iran, en thérapeutique, comme laxatif ou purgatif.

\section{CONDITIONS EXPÉRIMENTALES}

\section{- Irradiation}

L'irradiation des animaux (rats mâles de souche Wistar, agés de trois mois et pesant environ 250 grammes) est réalisée à l'aide d'une source de cobalt 60 de $2000 \mathrm{Ci}$. Le débit d'exposition est de 25 R.min. ${ }^{-1}$. Les rats sont placés dans des boîtes cylindriques, en plexiglass, tournant autour de leur axe de sorte que l'irradiation soit totale et homogène. La mesure de l'exposition est faite à l'aide d'une chambre d'ionisation et le calcul de la dose absorbée a nécessité une dosimétrie, sur cadavre de rat, par la méthode au sulfate ferreux et par chambre d'ionisation.

Travail effectué dans les laboratoires du Département de la Protection Sanitaire, Centre d'Etudes Nucléaires de Fontenay-aux-Roses, B.P. no 6, (92) Fontenay-aux-Roses.

* Détaché du Centre de Recherches du Service de Santé des Armées au Commissariat à l'Energie Atomique.

** Du Centre d'Etudes Nucléaires, de l'Université de Téhéran. 


\section{- Administration du Shir-Kbecht}

Le Shir-Khecht est administré aux animaux soit par voie orale, soit par voie intrapéritonéale, soit par les deux voies à la fois, avant l'irradiation.

La forme employée est soit le produit brut, soit la dissolution aqueuse ou l'hydrolysat, avec ou sans purification par dialyse.

Les quantités utilisées, ramenées au produit brut sont de 0,5 et $\mathrm{r}, 5 \mathrm{~g} / \mathrm{kg}$ de poids, en injection intrapéritonéale (l'injection est effectuée une demie heure avant l'irradiation) et de $0,5 \mathrm{~g} / \mathrm{kg} / \mathrm{jour}$, par voie orale.

Les témoins reçoivent, en injection intrapéritonéale ou par voie orale, un volume de sérum physiologique égal à celui qui a été utilisé pour les solutions testés.

Tous les groupes, y compris celui des témoins, sont formés de 20 animaux.

\section{RÉSultats}

L'activité protectrice éventuelle du Shir-Khecht a été recherchée par l'étude des courbes de survie, après irradiation à dose létale, des animaux traités et non traités.

Quelle que soit la forme utilisée, les courbes relatives aux animaux traités par le Shir-Khecht présentent une différence sensible par rapport aux courbes traduisant la survie des animaux témoins.

Les figures $\mathrm{I}, 2$ et 3 montrent les résultats obtenus, après irradiation à 800 , 850 et 950 rads des témoins et d'un groupe ayant reçu une injection intrapéritonéale de Shir-Khecht sous forme d'hydrolysat.

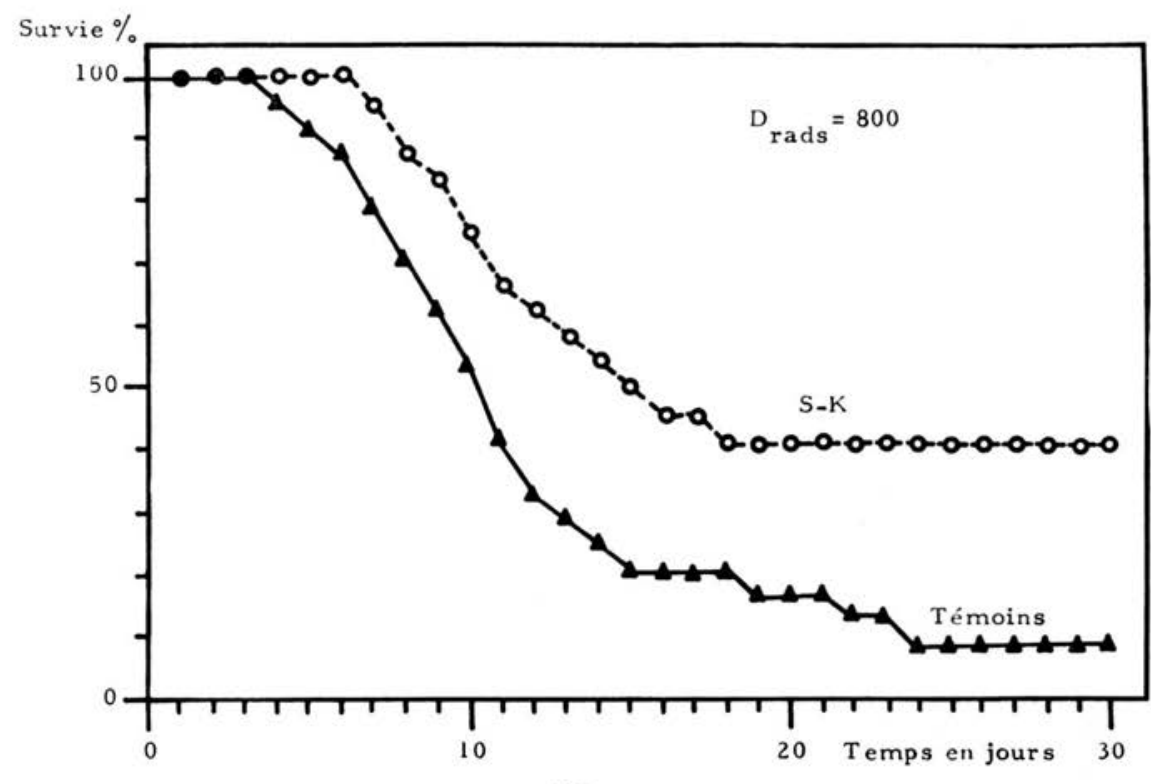

FIG. I. 
Survie \%

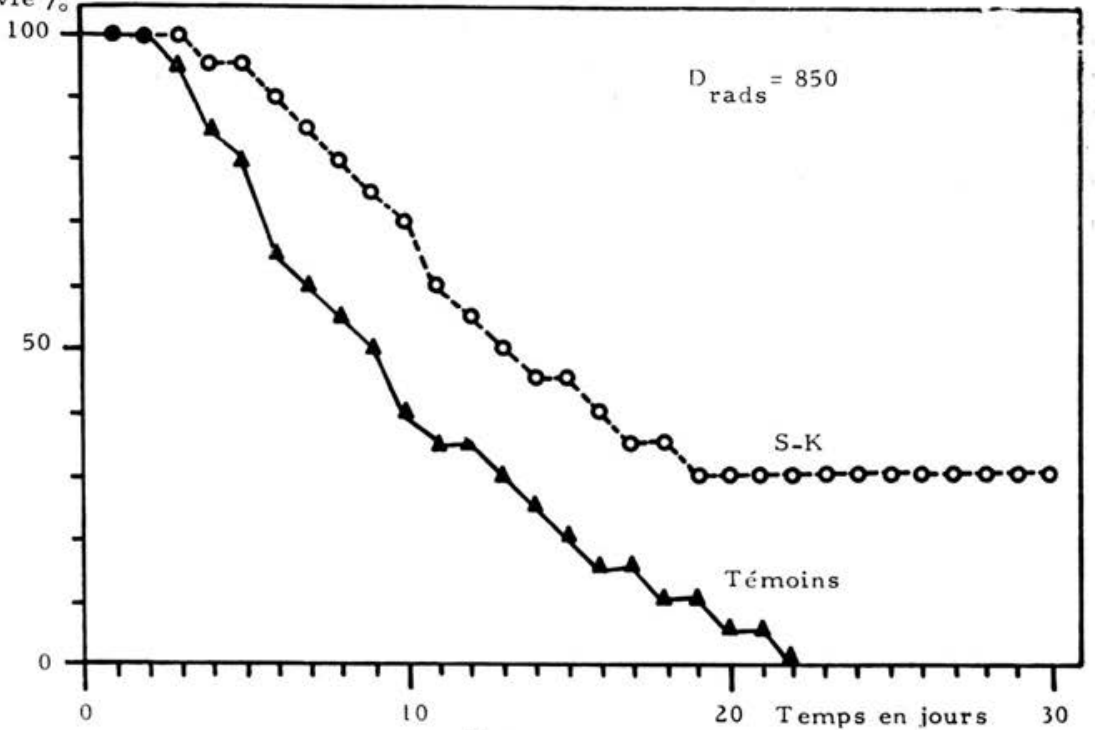

FIG. 2.

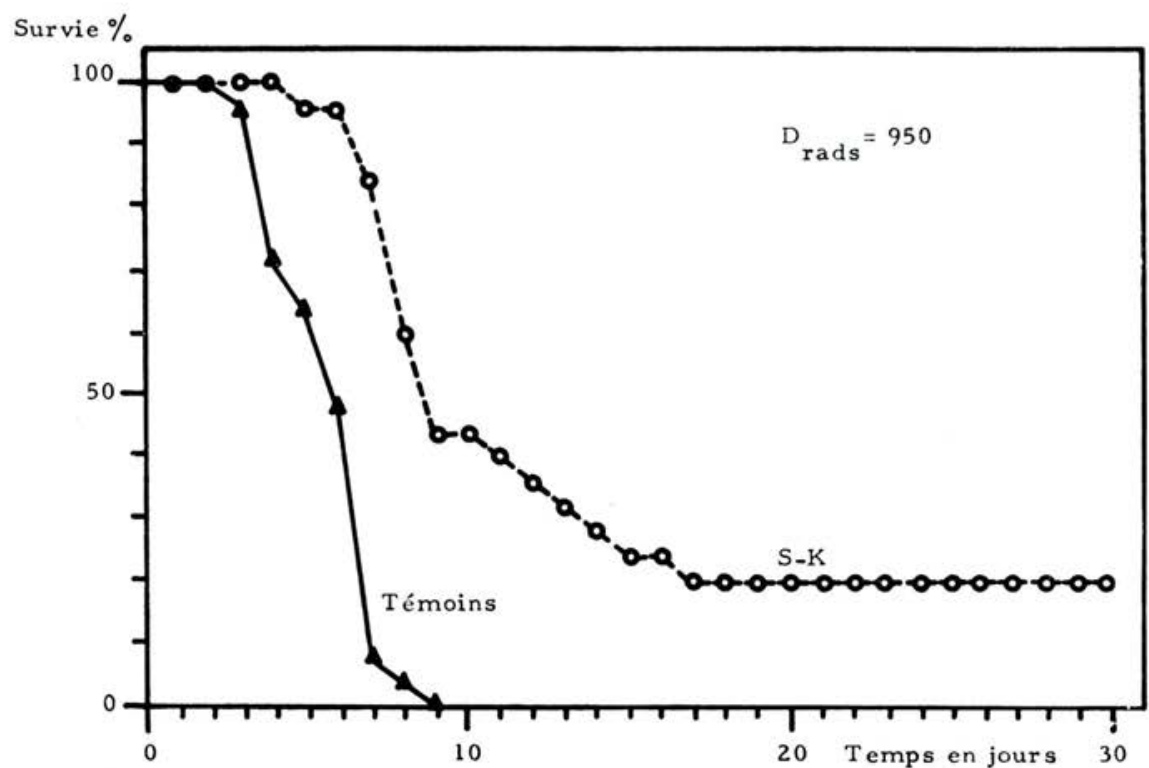

Fig. 3. 
Le Shir-Khecht est actif par voie orale. Les courbes de la figure 4 le montrent d'une façon nette. Ces courbes représentent le pourcentage de survie, en fonction du temps, après irradiation à 850 rads, des animaux ayant absorbé par voie orale $0,5 \mathrm{~g} / \mathrm{kg} /$ jour de Shir-Khecht pendant les ro jours qui précèdent l'irradiation, soit, au total, $1,25 \mathrm{~g}$ de Shir-Khecht, pour un rat de $250 \mathrm{~g}$. La forme utilisée a été le produit brut purifié par dialyse contre une solution de chlorure de sodium à $6 \mathrm{~g}$ par litre.

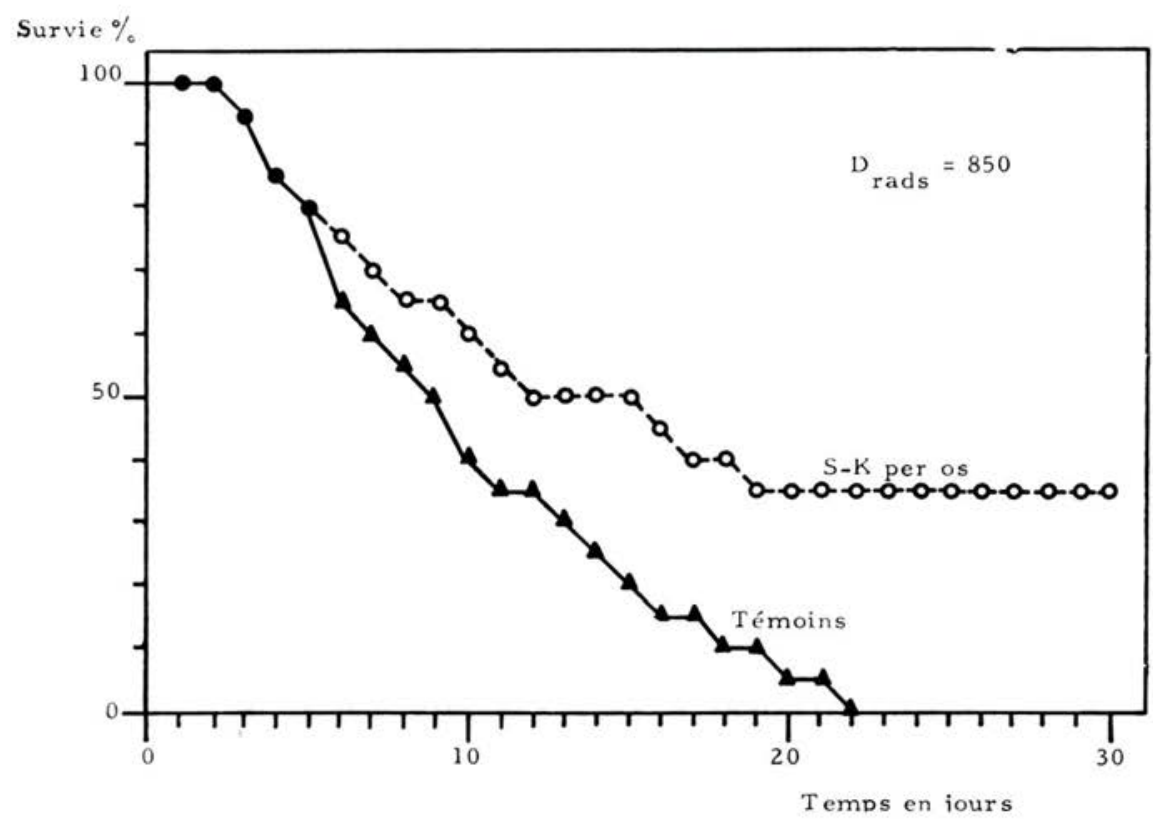

FIG. 4.

Le produit brut purifié par dialyse donne de bons résultats lorsqu'il est administré par voie orale (cf. fig. 4) ou par voie intrapéritonéale ( $c f$. fig. s) mais les résultats sont encore meilleurs si l'on utilise, de manière cumulative, les deux voies d'introduction. Les courbes de la figure s illustrent ces observations. Dans le dernier cas on obtient, en effet, une survie de $50 \%$ des animaux traités par le Shir-Khecht, irradiés à dose létale 100 .

L'effet radioprotecteur du Shir-Khecht est également observable à dose sublétale si l'on emploie une méthode biochimique d'examen de l'activité radioprotectrice. L'excrétion urinaire cumulée du potassium a été utilisée comme test (MA). On peut observer sur la courbe de la figure 6 relative aux animaux traités, la variation de pente qui se produit le quatrième jour, pente dont la valeur tend à se rapprocher de celle de la courbe relative aux animaux non irradiés. 
Survie \%

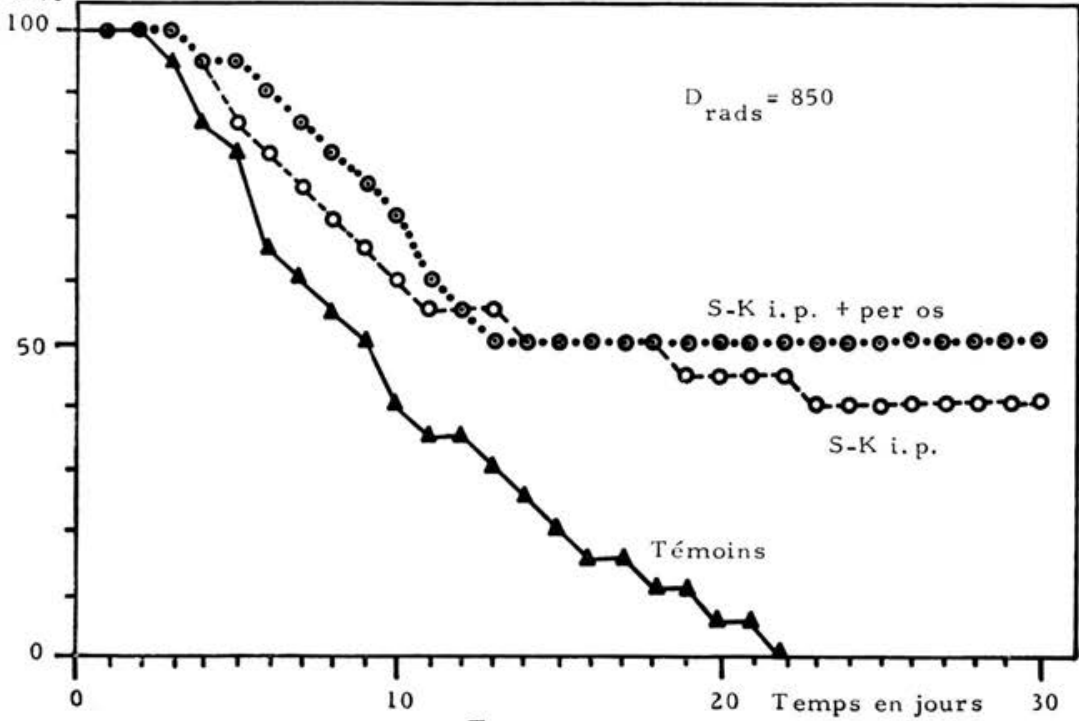

FIG. 5.

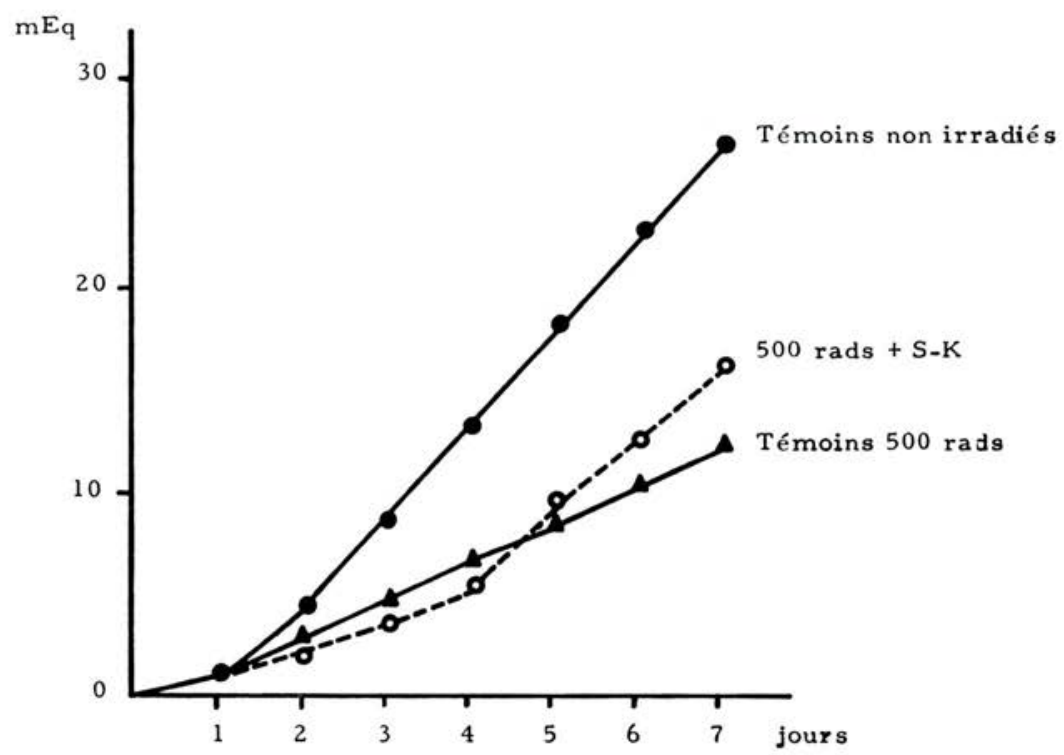

FIG. 6.

Excrétion urinaire cumulée de potassium 


\section{Conclusion}

Le Shir-Khecht présente de réelles propriétés radioprotectrices. Il n'est pas toxique et est actif par voie orale. Le produit brut purifié par dialyse permet d'obtenir une survie de $50 \%$ chez le rat irradié à dose létale $100 / 30$ jours. La purification du produit brut devrait permettre l'obtention d'un composé dosé d'un pouvoir radioprotecteur remarquable.

\section{BIBLIOGRAPHIE}

MARBLÉ G. Etude des variations des paramètres biochimiques après irradiation. Mémoire $\mathrm{n}^{\circ} 4$, ${ }^{\text {er }}$ Congrès International de l'I.R.P.A., Rome, septembre 1966.

Moghndam, S. Mannes de Perse. Thèse de Doctorat en Pharmacie, Paris, 1930.

PArsA, A. Flore d Iran. Ministère de 1 Education Nationale, Téhéran, Iran, I948-1958.

Rouhanizadeh, N. Effets de l'irradiation locale sur les testicules de rats normaux et protégés.

Thèse de D.E.A. Faculté des Sciences, Téhéran, Iran, 1964. 